University of Nebraska - Lincoln

DigitalCommons@University of Nebraska - Lincoln

2012

\title{
SIX2 and CITED1, markers of nephronic progenitor self-renewal, remain active in primitive elements of Wilms' tumor
}

\author{
Andrew J. Murphy \\ Monroe Carell Jr Children's Hospital at Vanderbilt, andrew.j.murphy@vanderbilt.edu \\ Janene Pierce \\ Monroe Carell Jr Children's Hospital at Vanderbilt \\ Christian de Caestecker \\ Monroe Carell Jr Children's Hospital at Vanderbilt \\ Chase Taylor \\ Monroe Carell Jr Children's Hospital at Vanderbilt \\ James R. Anderson \\ University of Nebraska Medical Center \\ See next page for additional authors
}

Follow this and additional works at: https://digitalcommons.unl.edu/publichealthresources

Murphy, Andrew J.; Pierce, Janene; de Caestecker, Christian; Taylor, Chase; Anderson, James R.; Perantoni, Alan O.; de Caestecker, Mark P.; and Lovvorn, Harold N. III, "SIX2 and CITED1, markers of nephronic progenitor self-renewal, remain active in primitive elements of Wilms' tumor" (2012). Public Health Resources. 278.

https://digitalcommons.unl.edu/publichealthresources/278

This Article is brought to you for free and open access by the Public Health Resources at DigitalCommons@University of Nebraska - Lincoln. It has been accepted for inclusion in Public Health Resources by an authorized administrator of DigitalCommons@University of Nebraska - Lincoln. 


\section{Authors}

Andrew J. Murphy, Janene Pierce, Christian de Caestecker, Chase Taylor, James R. Anderson, Alan O. Perantoni, Mark P. de Caestecker, and Harold N. Lovvorn III 


\title{
SIX2 and CITED1, markers of nephronic progenitor self-renewal, remain active in primitive elements of Wilms' tumor ${ }^{2}$, 抎败
}

\author{
Andrew J. Murphy ${ }^{\mathrm{a}, *}$, Janene Pierce ${ }^{\mathrm{a}}$, Christian de Caestecker ${ }^{\mathrm{a}}$, \\ Chase Taylor $^{a}$, James R. Anderson ${ }^{b}$, Alan 0. Perantoni ${ }^{c}$, \\ Mark P. de Caestecker ${ }^{d}$, Harold N. Lovvorn III ${ }^{\text {a }}$
}

${ }^{\mathrm{a} D e p a r t m e n t ~ o f ~ P e d i a t r i c ~ S u r g e r y, ~ M o n r o e ~ C a r e l l ~ J r ~ C h i l d r e n ' s ~ H o s p i t a l ~ a t ~ V a n d e r b i l t, ~ N a s h v i l l e, ~ T e n n e s s e e ~}$
${ }^{\mathrm{b}}$ Department of Biostatistics, Children's Oncology Group and University of Nebraska Medical Center, Omaha, Nebraska
${ }^{\mathrm{c} C a n c e r}$ and Developmental Biology Laboratory, Center for Cancer Research, National Cancer Institute, Frederick, Maryland
${ }^{\mathrm{d} D e p a r t m e n t s ~ o f ~ M e d i c i n e, ~ C e l l ~ a n d ~ D e v e l o p m e n t a l ~ B i o l o g y, ~ V a n d e r b i l t ~ U n i v e r s i t y ~ M e d i c a l ~ C e n t e r, ~ N a s h v i l l e, ~ T e n n e s s e e ~}$

Received 3 March 2012; accepted 6 March 2012

\author{
Key words: \\ SIX2; \\ CITED1; \\ Wilms' tumor; \\ Embryonic kidney
}

\begin{abstract}
Purpose: SIX2 and CITED1 are transcriptional regulators that specify self-renewing nephronic progenitor cells of the embryonic kidney. We hypothesized that SIX2, which promotes and maintains this stem cell population, and CITED1 remain active in Wilms' tumor (WT).

Methods: To evaluate expression domains and the pathogenic significance of SIX2 and CITED1 across WT, the Children's Oncology Group provided 40 WT specimens of stages I to IV ( $\mathrm{n}=10$ per stage), which were enriched for unfavorable histology $(\mathrm{n}=20)$ and treatment failure (relapse or death, $\mathrm{n}=20$ ). SIX2 and CITED1 protein expression was evaluated qualitatively (immunohistochemistry) and quantitatively (Western blot, or WB). Gene transcription was estimated using quantitative real-time polymerase chain reaction (qRT-PCR).

Results: SIX2 was visualized by immunohistochemistry in $36(94.7 \%)$ of 38 specimens. Protein and messenger RNA expression of SIX2 were quantitatively similar across all stages of disease $(P=.48 \mathrm{WB}$; $P=0.38 \mathrm{qPCR})$, in favorable or unfavorable histology $(P=0.51 \mathrm{WB} ; P=0.58 \mathrm{qPCR})$, and in treatment failure or success $(P=0.86 \mathrm{WB} ; P=0.49$ qPCR). Although CITED1 expression paralleled SIX2 qualitatively, no quantitative correlation between SIX2 and CITED1 expression was observed (Spearman correlation coefficient, $0.28 ; P=0.08$ ). As in the fetal kidney, overlapping, but also distinct, WT cellular expression domains were observed between SIX2 and CITED1.

Conclusion: SIX2 and CITED1 remain active across all disease characteristics of WT. Activity of these genes in WT potentially identifies a population of self-renewing cancer cells that exhibit an embryonic,
\end{abstract}

\footnotetext{
it Sources of Support: This work was supported by the Section of Surgical Sciences and the Ingram Cancer Center of the Vanderbilt University Medical Center, by the National Cancer Institute grant 5T32CA106183-06A1 (AJM), and by NCI grant 4R00CA135695-03 (HNL).

is Conflict of Interest Statement: The authors have no conflicts of interest to report.

* Corresponding author. Tel.: +1 615936 1050; fax: +1 6159361046.

E-mail address: andrew.j.murphy@vanderbilt.edu (A.J. Murphy).
} 
stemlike phenotype. Taken together, these transcriptional regulators may be fundamental to WT cellular self-renewal and may represent targets for novel therapies that promote terminal differentiation.

(C) 2012 Elsevier Inc. All rights reserved.

Wilms' tumor (WT) is the most common kidney cancer of childhood and is thought to arise from arrested epithelial differentiation of nephronic progenitor cells of the embryonic kidney [1,2]. Normal nephron development results from reciprocal inductive signaling between the ureteric bud tip epithelium and the surrounding condensing metanephric mesenchyme (MM) [3]. The condensing or cap mesenchyme must balance the dual fates of either epithelial differentiation into functional nephrons or selfrenewal, which maintains the pool of nephronic progenitors until kidney maturation is complete [4]. During kidney development, expression of 2 transcriptional regulators, SIX2 and CITED1, specifies the self-renewing population of nephronic progenitor cells within the condensing MM [4]. SIX2 has been shown to be a direct regulator of nephronic progenitor self-renewal and has been shown to suppress epithelial differentiation and promote maintenance of the MM [5]. Fate mapping of CITED1- and SIX2-positive cells within the MM was used to discover the self-renewing capacity of the MM $[5,6]$. In separate studies, however, CITED1 null mice did not exhibit altered nephrogenesis, signifying that CITED1 expression is not solely responsible for maintenance of nephronic progenitors [7]. In contrast, SIX2 null mice exhibit marked metanephric hypoplasia, indicating that SIX2 is required for progenitor cell self-renewal $[5,8]$.

Wilms' tumor caricatures the classic triphasic histology of the embryonic kidney (blastema, epithelia, stroma), with the blastemal compartment representing the neoplastic analogue of the MM in development [9]. Clarifying signaling pathways, which function as gatekeepers of progenitor self-renewal and mesenchymal to epithelial transition, may yield clues as how to promote terminal differentiation of WT, lessons potentially applicable to other embryonal tumors. Importantly, although SIX2 and CITED1 become inactive in the earliest phases of epithelial and nephronic maturation and are "off" in the mature kidney, we have previously shown that CITED1 remains active in WT and richly labels WT blastema [10]. In those studies, increased CITED1 expression was associated with stage IV disease in favorable histology WT [10]. Mechanistically, we have shown that overexpression of wild-type CITED1 is proproliferative in the malignant context and that deletion of the CITED1 transactivation domain attenuates Wilms' tumorigenesis [11]. However, the functional and pathogenic significance of SIX2 in WT development and disease progression has yet to be defined. In this study, we aimed to characterize SIX2 expression in the context of CITED1 and also to determine if expression patterns of either gene had pathogenic features in favorable and unfavorable histology WT.

\section{Methods}

\subsection{Acquisition of tissue specimens}

The institutional review board of Vanderbilt University Medical Center approved all studies involving human tumor and embryonic kidney specimens (institutional review board no. 020888). To explore SIX2 and CITED1 activity in WT and their association with adverse clinical features, the Children's Oncology Group provided 40 corresponding paraffin-embedded and fresh-frozen WT specimens from stages I to IV of disease ( $\mathrm{n}=10$ per stage), which were enriched for unfavorable histology $(\mathrm{n}=20)$, and treatment failure (disease relapse or death, $n=20$ ). Specimens were deidentified, and the investigative team was blinded to all clinical details of patient samples until after data analysis had been completed.

Discarded human fetal kidneys (having gestational ages 16, 20, and 24 weeks) were procured from therapeutic abortuses (Advanced Bioscience Resources, Inc, Alameda, CA). Specimens were shipped overnight in sterile media on ice and fixed immediately on arrival in 10\% buffered formalin. Mouse fetal kidneys (MFKs) from gestational ages e13 to e18.5 (days postconception) were similarly processed and used for experimental analysis.

\subsection{Immunohistochemistry}

We immunostained the aforementioned WT and fetal kidney specimens to characterize the cellular expression domains of SIX2 and CITED1 in the malignant and embryonic contexts. All tissue samples were subjected to heat-induced epitope retrieval in $10 \mathrm{mM}$ citrate buffer. As described previously [10], these $5-\mu \mathrm{m}$ sections were incubated in affinity-purified rabbit anti-CITED1 (1:50 dilution; Lab Vision Corp, Fremont, CA) or rabbit antiSIX2 (1:25 dilution; US Biological Corp, Marblehead, MA) antibodies for 1 hour at room temperature. Goat antirabbit secondary antibody (1:200 dilution; Santa Cruz Biotechnology, Santa Cruz, CA) was applied to tissues at room temperature for 45 minutes. Tissues were visualized with either a Vectastain $\mathrm{ABC}$ kit (Vector Laboratories, Burlingame, CA) or DAKO Envision kit (DAKOCytomation, Carpinteria, CA). Because SIX2 has not been 
previously characterized in WT by immunohistochemistry, we validated our observations using a second anti-SIX2 antibody (1:150 dilution, mouse monoclonal; Abnova, Walnut, CA).

\subsection{Immunofluorescence}

To colocalize the distribution of SIX2 and CITED1 expression among cell populations of human WT and fetal kidney specimens, we performed double immunofluorescence. Five-micrometer paraffin-embedded sections were deparaffinized and rehydrated. Sections were subjected to heat-induced epitope retrieval as described above. Endogenous tissue peroxidase activity was quenched using 3\% hydrogen peroxide in methanol. Human fetal kidney and WT sections were blocked using 10\% goat serum in phosphatebuffered saline (PBS) for 1 hour at room temperature. These sections were then incubated in affinity-purified rabbit antiCITED1 (1:2500 dilution; Lab Vision Corp) and mouse antiSIX2 (1:5000 dilution; Abnova) in 10\% goat serum and PBS overnight at $4^{\circ} \mathrm{C}$. DAKO Envision antimouse horseradish peroxidase (HRP) polymer (DAKOCytomation) was added for 30 minutes at room temperature. For SIX2 signal amplification, Tyramide Signal Amplification (TSA) plus Fluorescein isothiocyanate (FITC) fluorophore was diluted in TSA amplification diluent and placed on sections for 10 minutes at room temperature (1:200 dilution; Perkin Elmer, Waltham, MA). Unconjugated horseradish peroxidase was quenched using $6 \%$ hydrogen peroxide in PBS for 15 minutes at room temperature. DAKO Envision antirabbit HRP polymer (DAKOCytomation) was added for 30 minutes at room temperature. For CITED1 signal amplification, TSA plus Cy3 flurophore (Perkin Elmer) was diluted in TSA amplification diluent and placed on tissues for 10 minutes at room temperature. Nuclear counterstain was performed using DAPI (1:50,000 dilution in PBS; Invitrogen, Eugene, OR) for 2 minutes at room temperature.

For MFKs, sections were similarly deparaffinized, rehydrated, subjected to heat-induced epitope retrieval, and quenched using hydrogen peroxide. Mouse fetal kidney specimens were blocked using mouse-on-mouse block (Vector Laboratories, Burlingame, CA) with $0.5 \%$ hydrogen peroxide for 1 hour at room temperature. Sections were then incubated in rabbit anti-CITED1 (1:100; Lab Vision Corp) and mouse anti-SIX2 (1:100; Abnova) with mouse-onmouse block, $10 \%$ goat serum, and $0.5 \%$ hydrogen peroxide in PBS overnight at $4{ }^{\circ} \mathrm{C}$. Antimouse HRP (1:750 dilution; KPL, Gaithersburg, MD) was added for 40 minutes at room temperature. For SIX2 signal amplification, TSA plus FITC fluorophore was diluted in TSA amplification diluent and placed on sections for 9 minutes at room temperature (1:200 dilution; Perkin Elmer). For visualization of CITED1, antirabbit DyLight 549 secondary antibody was added (1:600 dilution; Jackson Immunoresearch, West Grove, PA) for 2 hours at room temperature.

\subsection{Western blots}

To quantify differences in protein expression across various disease characteristics of WT, tissue lysates were prepared by homogenizing snap-frozen WT specimens in lysis buffer containing protease and phosphatase inhibitors $(150 \mathrm{mM} \mathrm{NaCl}, 1 \%$ Triton $\mathrm{X}, 5 \mathrm{mM}$ EDTA, $50 \mathrm{mM}$ Hepes, $2.1 \mathrm{mM}$ lepeptin, $0.15 \mathrm{mM}$ aprotinin, $1 \mathrm{mM}$ $\mathrm{Na}_{2} \mathrm{VO}_{3}, 50 \mathrm{mM} \mathrm{NaF}$ ). Resulting protein tissue lysates were quantified using a SpectraMax M5 spectrophotometer (Molecular Devices, Sunnyvale, CA). Two hundred micrograms of protein lysate for CITED1 and $100 \mu \mathrm{g}$ of lysate for SIX2 were heat-denatured and run on $10 \%$ BisTris NuPAGE electrophoresis gels in $1 \times$ MOPS NuPAGE SDS running buffer at $200 \mathrm{~V}$ for 45 minutes at RT (Invitrogen). Proteins were then transferred to PVDF membranes in $1 \times$ NuPAGE transfer buffer (Invitrogen) at $30 \mathrm{~V}$ for 1 hour at $4^{\circ} \mathrm{C}$. Immunoblots were performed using affinity-purified rabbit anti-CITED1 (1:1000 dilution; Lab Vision Corp), rabbit anti-SIX2 (1:1000 dilution; US Biological Corp), and mouse anti- $\beta$-actin (1:5000 dilution; Sigma Chemical Co, St. Louis, MO). Antibodyantigen complexes were visualized with HRP-conjugated secondary antibodies (goat antirabbit FC fragment specific antibody 1:10000 dilution; Jackson Immunoresearch, and goat antimouse secondary 1:1000 dilution; KPL). Specificity of SIX2 bands was confirmed using a second SIX2 antibody (1:5000 dilution, mouse monoclonal; Abnova). Immunoblots were developed using Pierce ECL Western blotting substrates (Thermo Scientific, Rockford, IL) and visualized on $\mathrm{x}$-ray radiograph. Bands were identified and quantified using Photoshop software (Adobe, San Jose, CA). Results were normalized to $\beta$-actin. MCF7, a breast cancer cell line known to express CITED1, was used for a CITED1-positive control. COS cell lysate, a transformed monkey kidney cell line, was used for a negative control. SIX2-positive controls were e18.5 MFKs and VUWT, a WT also determined to have high SIX2 expression by immunohistochemistry and quantitative real-time polymerase chain reaction (qRT-PCR). SIX2 negative controls were COS cell lysate and a congenital mesoblastic nephroma.

\subsection{Quantitative qRT-PCR}

To evaluate differences in gene transcription of $S I X 2$ and CITED1 across disease characteristics of WT, total RNA was isolated and purified from snap-frozen WT tissues using RNAzol (Tel-Test Inc, Friendswood, TX) and RNeasy mini kits (Qiagen, Germantown, MD). Isolated RNA was quantified using a SpectraMax M5 UV spectrophotometer (Molecular Devices). Reverse transcription of 3- $\mu \mathrm{g}$ RNA was performed using Superscript II reverse transcriptase (Invitrogen) and oligo(dT) primers (Applied Biosystems, Foster City, CA) to synthesize complementary DNA suitable for analysis by quantitative 
qRT-PCR (Bio-Rad iCycler; Bio-Rad, Hercules, CA) using iQ SYBR Green Super Mix (Bio-Rad). Primers used are included below:

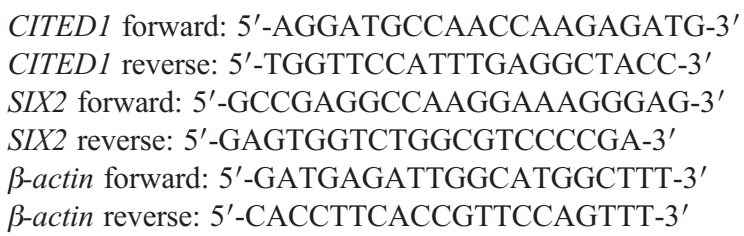

Changes in messenger RNA (mRNA) expression were determined by comparison of sample cycle threshold values against a standard curve generated using pooled sample or plasmid complementary DNA. Results were normalized to $\beta$-actin level and compared statistically.

\subsection{Statistical analysis}

One aim of this study was to determine whether quantities of SIX2 or CITED1 expression correlated with patient or disease variables including age, sex, stage of disease, favorable or unfavorable histology, and treatment failure (disease relapse or death). We also questioned whether tumors with immunohistochemical detection of SIX2 in a pattern reminiscent of the embryonic kidney correlated with the above variables. Results from the above analyses were compared among subsets of patients using the 2-sample Wilcoxon's test (or the Kruskal-Wallis test for more than 2 groups). Nonparametric comparisons were performed because the distribution of study variables appeared to be long tailed. To determine correlation between qPCR and Western blot expression levels of CITED1 or SIX2, a nonparametric measure of correlation was used (Spearman correlation coefficient). Calculations were performed using the SAS statistical software package (SAS, Cary, NC). Statistical significance was set at $P<.05$.

\section{Results}

\subsection{Immunohistochemistry}

In 36 of 38 WT specimens suitable for examination, SIX2 was detected by immunohistochemistry (94.7\%) (Fig. 1). In 24 specimens $(63.2 \%)$, staining was restricted to the blastemal compartment only. Nine specimens (23.7\%) showed concomitant blastemal and epithelial SIX2 immunopositivity, and 3 specimens (7.9\%) showed only epithelial positivity. Regardless of blastemal or epithelial detection, SIX2 was localized exclusively to the nucleus.

Expression of SIX2 was detected across all patient and disease characteristics evaluated, including age, sex, stage, histology, and treatment failure. Of the 38 specimens analyzed by immunohistochemistry, 10 (26.3\%) exhibited a SIX2 immunostaining pattern reminiscent of that observed in the embryonic kidney (Fig. 1A and B;
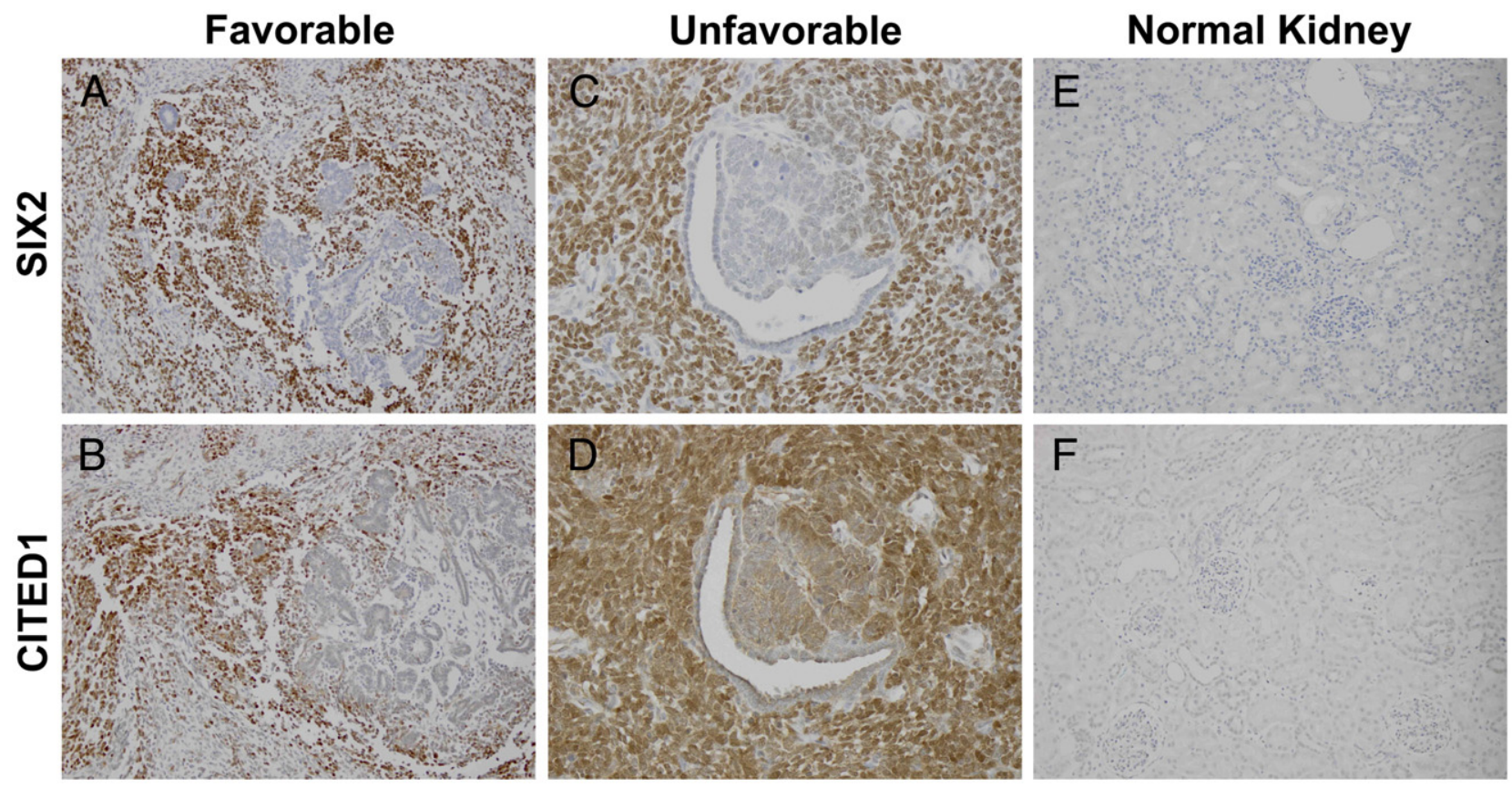

Fig. 1 Serial sections from a favorable histology WT show blastemal immunopositivity for SIX2 (A) and CITED1 (B). Epithelia and stroma are immunonegative, similar to the embryonic kidney staining pattern (original magnification $\times 20$ ). Serial sections from unfavorable histology WT with similar overlap in blastemal expression of SIX2 (C) and CITED1 (D). Areas surrounding epithelial differentiation are weakly CITED1 positive, but SIX2 negative, demonstrating divergent staining patterns unique to a proportion of tumors examined (original magnification $\times 40$ ). Normal kidney controls show immunonegativity for SIX2 (E) and CITED1 (F) (original magnification $\times 20$ ). 
blastema positive, epithelia negative, stroma negative). However, this staining pattern did not specifically associate with age $(P=.11)$, sex $(P=1.00)$, stage of disease $(P=$ $.87)$, favorable or unfavorable histology $(P=.27)$, or treatment failure or success $(P=.15)$. For this analysis, we considered the WT blastema analogous to the cap mesenchyme of the embryonic kidney.

CITED1 immunostaining was detected in $33(86.8 \%)$ of 38 WT specimens examined. Twelve tumors $(31.6 \%)$ exhibited solely blastemal CITED1 immunostaining, whereas $16(42.1 \%)$ showed both blastemal and epithelial CITED1 positivity. Unlike SIX2, no tumors were detected with exclusively epithelial CITED1 immunostaining. When serial tumor sections were examined, immunodetection of SIX2 and CITED1 appeared to overlap in similar tumor histologic compartments and regions, but some tumors showed unique expression of one or the other protein between both individual cells and larger tumor compartments (Fig. 1). Neither CITED1 nor SIX2 was detected by immunohistochemistry in normal kidney controls (Fig. 1E and F).

Immunohistochemical detection of SIX2 and CITED1 in the e18.5 MFK was limited to the cap mesenchyme surrounding the ureteric bud tips (Fig. 2A and B). A population of SIX2+/CITED1- cells within the ventral cap mesenchyme and in adjacent pretubular aggregates was suggested by immunohistochemistry (Fig. 2A and B). To further investigate the relationship between subcellular localization and compartmental expression of SIX2 and CITED1 within the MM and WT, we colocalized these proteins using double immunofluorescence.

\subsection{Immunofluorescence colabeling studies}

Because SIX2 and CITED1 show both overlapping and differential expression domains in the MM and WT, we tested in which contexts these 2 proteins colocalized and whether any associations with disease characteristics were evident according to their differential expression or colocalization. Immunofluorescent colabeling of the e18.5 MFK shows expression of SIX2 and CITED1 in the dorsal cap mesenchyme surrounding the ureteric bud tip epithelia (Fig. 2). In this embryonic context, the subcellular localization of SIX2 is exclusively nuclear, whereas CITED1 is detected predominantly in the cytoplasm (Fig. 2C). Merged images of their respective expression domains label an identical cell population within the cap mesenchyme that expresses both SIX2 and CITED1; however, a distinct population at the border of the ventral cap mesenchyme and in pretubular aggregates appears to express only SIX2 but not CITED1 (Fig. 2C). This experiment was repeated using 16-week human fetal kidney specimens showing similar findings (Fig. 3).

Colabeling of both unfavorable and favorable histology WT shows SIX2 and CITED1 expression to be predominantly nuclear and within the blastemal compartment of tumors, although CITED1, and not SIX2, is concurrently detected in the cytosol (Fig. 4). This nuclear enrichment of CITED1 in malignant blastema is in marked contrast to its predominantly cytosolic localization in the embryonic kidney [10]. Among blastemal compartments, identical cells were shown to express both SIX2 and CITED1; however, rare blastemal cells, and even larger tumor
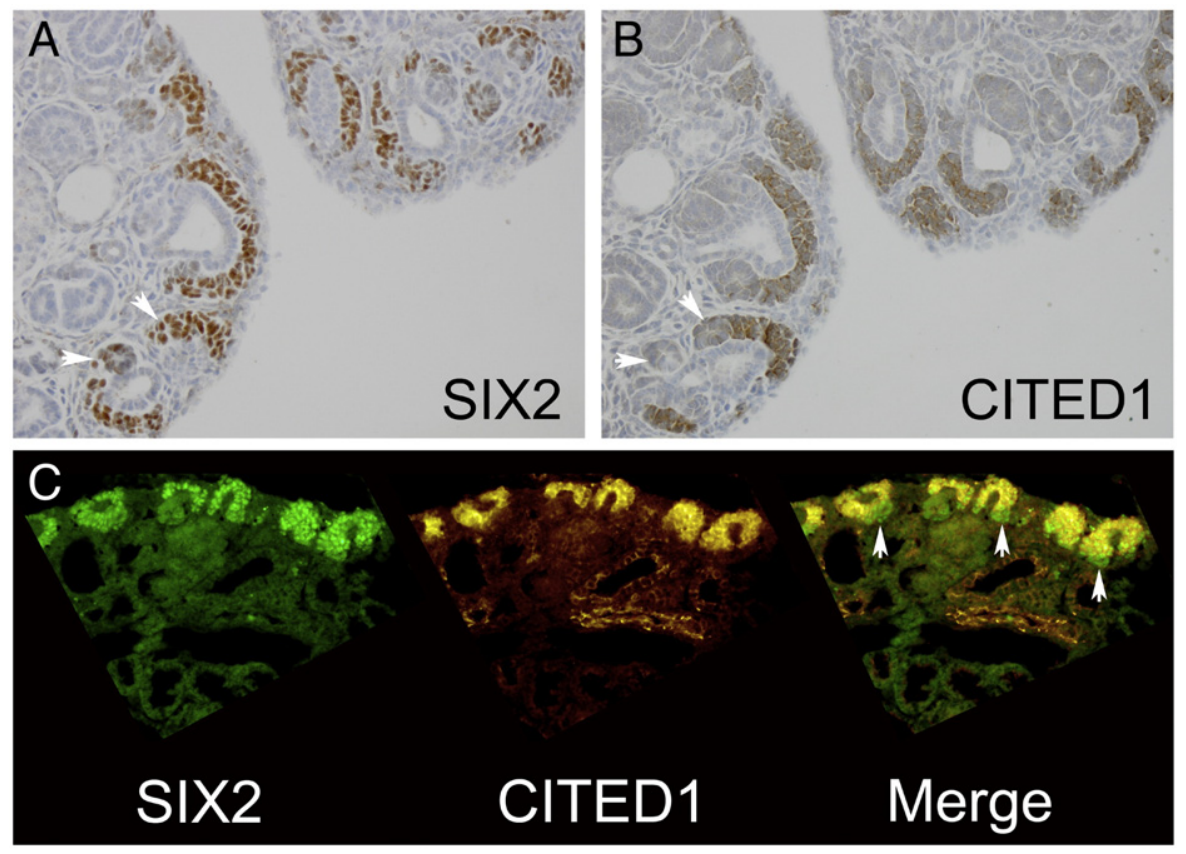

Fig. 2 Serial sections from e18.5 MFK show a population of cells in the ventral cap mesenchyme and in pretubular aggregates (arrowheads) that are SIX2 positive (A), but CITED1 negative (B) (original magnification $\times 20$ ). Double immunofluorescence for SIX2 and CITED1 on the same section (C) identifies this SIX2-positive, CITED1-negative population in the ventral cap mesenchyme (arrowheads) (original magnification $\times 20$ ). 


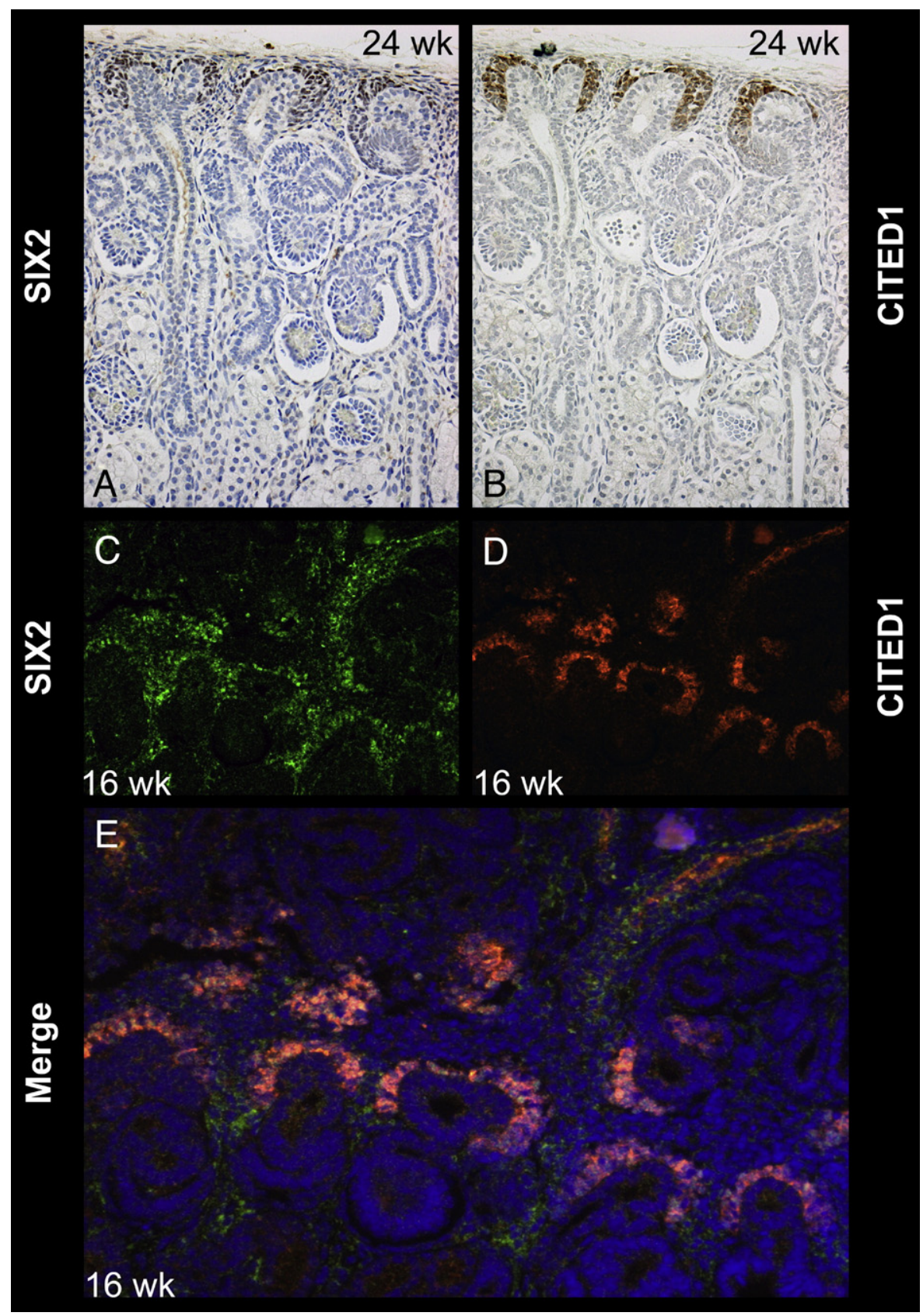

Fig. 3 Serial sections from a 24-week human fetal kidney show SIX2 (A) and CITED1 (B) immunopositivity corresponding to the cap mesenchyme (original magnification $\times 20$ ). Double immunofluorescence on a 16-week human fetal kidney demonstrates SIX2 (C) and CITED1 (D) positivity in the cap mesenchyme (E, merged image) (original magnification $\times 20$ ).

regions, were observed to express either one or the other protein (Fig. 4).

SIX2 expression in the developing kidney may be observed in pretubular aggregates (Fig. 2), which represent the earliest epithelial structure of the nephron but is not observed in more mature nephronic epithelia. In WT, certain primitive epithelial structures indeed expressed nuclear SIX2; however, unlike the embryonic kidney, CITED1 was also detected in primitive malignant epithelia and was predominantly cytosolic in these more differentiated structures (Fig. 4).

\subsection{Quantification of SIX2 and CITED1 expression}

By Western blot, SIX2 and CITED1 were detected broadly across all WT investigated and showed variable quantity of expression among samples (Fig. 5). SIX2 was visualized as a single band at $37 \mathrm{kd}$, and CITED1, as multiple bands from 25 to $32 \mathrm{kd}$, consistent with previously described variability in its molecular weight because of posttranslational modifications $[10,12]$. Both SIX2 and CITED1 were detected across all demographic and disease characteristics of WT, regardless of patient age, sex, tumor stage, favorable 
Favorable

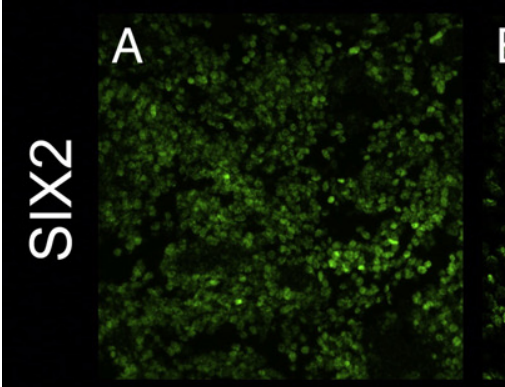

B

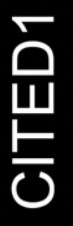

C

G

St

BI

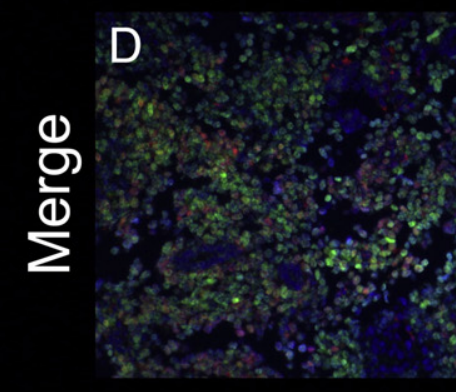

\section{E}

F

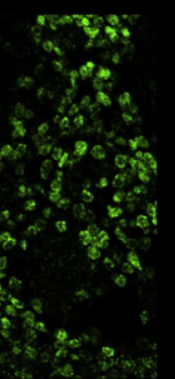

J

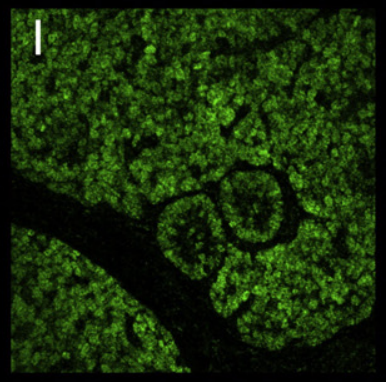

J

K

$\mathrm{BI}$
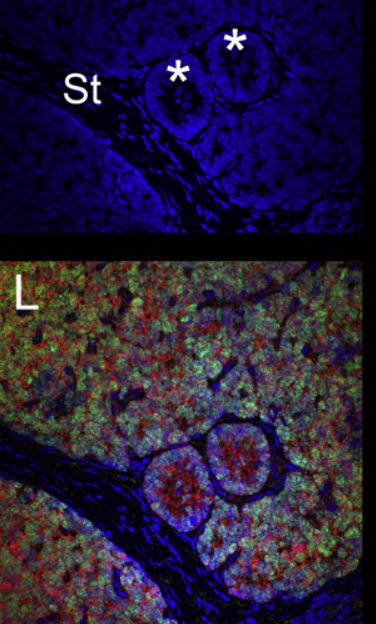

M

able

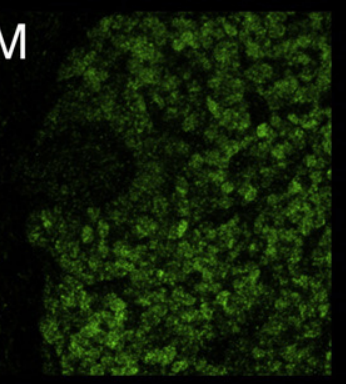

N

O

\section{Ep}

BI

St

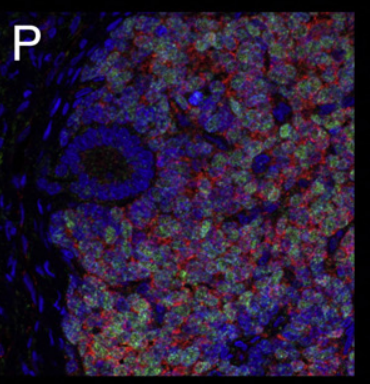

Fig. 4 Double immunofluorescence colocalizes SIX2 (A and E) and CITED1 (B and F) within the blastema of favorable histology WT. Individual cells within the tumor blastema are heterogeneous with respect to SIX2 and CITED1 intensity (D and H). An area of aggregated blastema is negative for SIX2 and CITED1 (G, asterisk; B1 indicates blastema, and St, stroma). Colocalization of SIX2 (I and M) and CITED1 ( $\mathrm{J}$ and $\mathrm{N}$ ) in unfavorable histology WT (L and P merged images). Areas of epithelial differentiation (K, asterisk) may be either positive for SIX2 or CITED1 (L) or negative $(\mathrm{O}, \mathrm{Ep})$ depending on the tumor or region within a given tumor (A-D, original magnification $\times 20$; E-P, original magnification $\times 40$ ).

or unfavorable histology, or treatment failure or success (Fig. 5A, B, and C; Table). Likewise, similar levels of SIX2 and CITED1 transcription were detected by qRTPCR across all WT regardless of patient or pathogenic characteristics (Table).

Given the temporal and spatial overlap between SIX2 and CITED1 both in kidney development and in WT, we used Western blot densitometry and qRT-PCR to quantify the correlation between respective expression levels across WT. Using a nonparametric measure of correlation (Spearman correlation coefficient), the correlation between SIX2 and CITED1 protein expression normalized to $\beta$-actin was 0.42 ; the $P$ value testing the null hypothesis that the variables were independent was .0081. Using qRT-PCR, the correlation between SIX2 and CITED 1 mRNA expression was 0.28 ; the $P$ value testing the null hypothesis that the values were independent was 0.08 . 

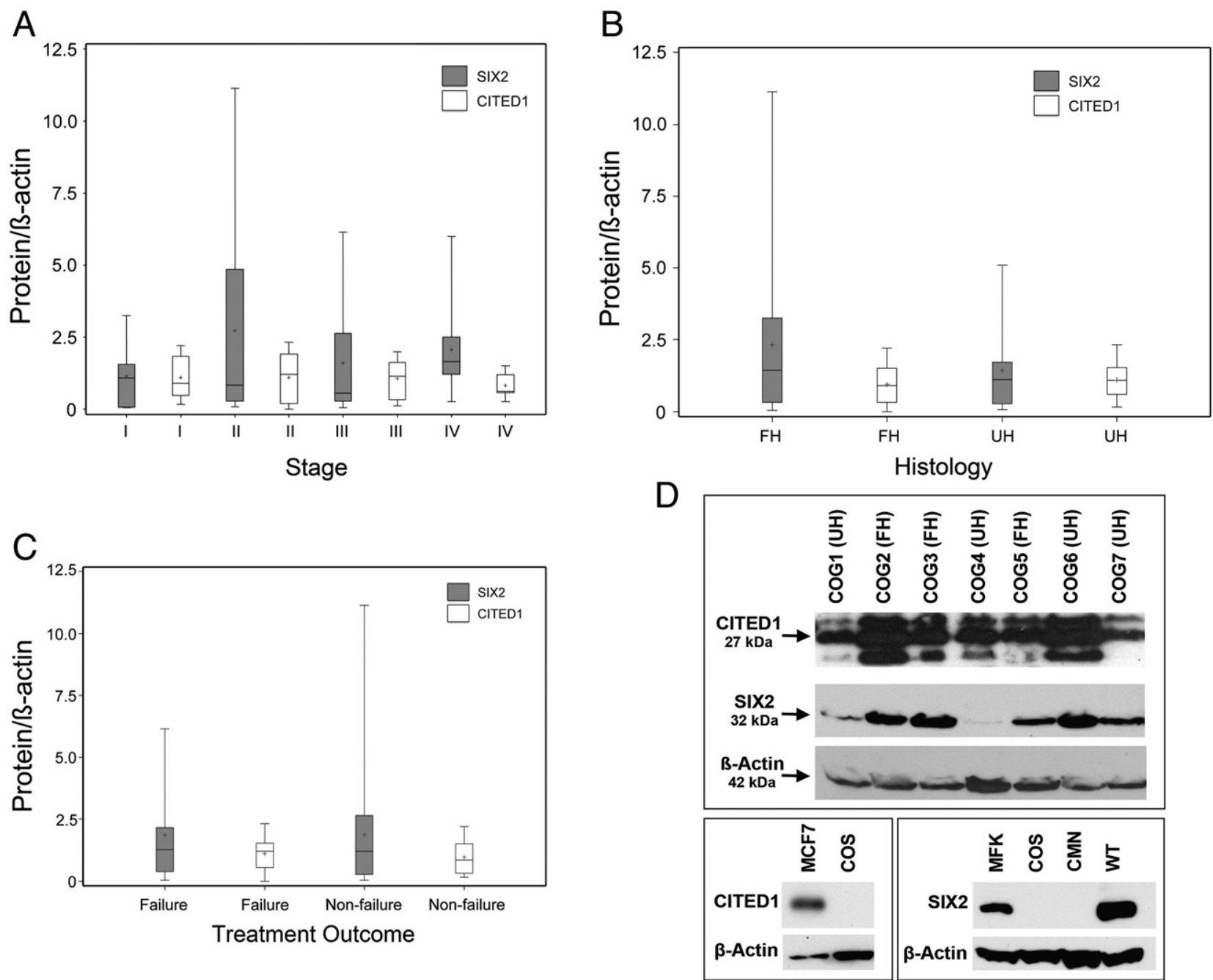

Fig. 5 Boxplot representation of SIX2 and CITED1 demonstrates ubiquitous expression across samples grouped by tumor stage (A), histology (B), and treatment outcome (C; failure = relapse or death). (D) Western blots for SIX2 and CITED1 in favorable histology (FH) and unfavorable histology $(\mathrm{UH}) \mathrm{WT}$ specimens confirm ubiquitous expression, yet variability among samples. The CITED1 band at $27 \mathrm{kd}$ was used for densitometry and ran at same molecular weight as MCF7 positive control. Lower panels depict positive and negative controls (MCF7 indicates MCF7 cell lysate; COS, COS cell lysate; MFK, e18.5 MFK; CMN, congenital mesoblastic nephroma; VUWT, Wilms' tumor).

\section{Discussion}

This study is the first to characterize SIX2 expression across a broad spectrum of WT. Our findings showed that the expression of SIX2 and CITED1, transcriptional regulators that specify self-renewing nephron progenitor cells in kidney development, was highly consistent across WT regardless of stage of disease, tumor histology, or treatment outcome. Paralleling the MM of kidney development [13], SIX2 and CITED1 were predominantly active in the blastemal compartment of WT and in overlapping cell populations, yet also showed independent expression domains within WT compartments and in subcellular localization. Although expression of these genes in WT did not associate with any specific disease or demographic characteristic, the broad detection of SIX2 and CITED1, genes that are normally inactive in differentiated nephrons of the embryonic and adult kidney, suggests that persistence or reactivation of embryonal transcriptional programs is fundamental to Wilms' tumorigenesis and, furthermore, implicates the condensing MM as the WT cell population of origin.

The principal aim of this study was to clarify the pathogenic significance of SIX2 across all stages and histologies of WT. We were surprised to uncover the nearly ubiquitous expression of this transcriptional regulator, critical to nephronic progenitor self-renewal, across all varieties of WT. Evidence is emerging to suggest that embryonal transcriptional programs and other genes expressed in the MM indeed remain active or are reactivated in WT, including WT1 [14], PAX2 [15], SIX1, EYA1, SALL2, HOXA11 [1], IGF2 [16], and CITED1 [10] genes. The critical nature of genes expressed in the MM is further underscored by the recent development of the first endogenous genetic mouse model of WT by WT1 ablation 
Table Median SIX2 and CITED1 expression by Western blot and qRT-PCR stratified according to patient demographics, WT stage, histology, and treatment outcome

\begin{tabular}{|c|c|c|c|c|c|c|c|c|c|}
\hline \multirow[t]{2}{*}{ Characteristic } & \multirow[t]{2}{*}{$\mathrm{n}$} & \multicolumn{4}{|c|}{ Median SIX2 normalized to $\beta$-actin } & \multicolumn{4}{|c|}{ Median CITED1 normalized to $\beta$-actin } \\
\hline & & Protein & $P$ & mRNA & $P$ & Protein & $P$ & mRNA & $P$ \\
\hline \multicolumn{10}{|l|}{ Age } \\
\hline $0-23$ mo & 11 & 0.84 & & 1.10 & & 0.48 & & .00178 & \\
\hline $24+\mathrm{mo}$ & 28 & 1.35 & .34 & 1.01 & .54 & 1.21 & .03 & .00352 & .08 \\
\hline \multicolumn{10}{|l|}{ Sex } \\
\hline Male & 19 & 1.21 & & 1.19 & & 0.91 & & .00311 & \\
\hline Female & 20 & 1.24 & .62 & 0.72 & .05 & 0.93 & .76 & .00317 & .46 \\
\hline \multicolumn{10}{|l|}{ Stage } \\
\hline I & 10 & 1.08 & & 0.94 & & 0.91 & & .00454 & \\
\hline II & 9 & 0.84 & & 1.18 & & 1.21 & & .00169 & \\
\hline III & 10 & 0.57 & & 0.73 & & 1.43 & & .00254 & \\
\hline IV & 10 & 1.66 & .48 & 1.28 & .38 & 0.63 & .86 & .00344 & .43 \\
\hline \multicolumn{10}{|l|}{ Histology } \\
\hline Favorable & 19 & 1.44 & & 0.90 & & 0.90 & & .00346 & \\
\hline Unfavorable (anaplasia) & 20 & 1.12 & .51 & 1.10 & .58 & 1.10 & .50 & .00253 & .88 \\
\hline \multicolumn{10}{|l|}{ Outcome } \\
\hline Failure (relapse or death) & 16 & 1.27 & & 1.09 & & 1.21 & & .00344 & \\
\hline Nonfailure & 23 & 1.21 & .86 & 1.02 & .49 & 0.86 & .49 & .00274 & .61 \\
\hline
\end{tabular}

and IGF2 overexpression [17]. Dysregulated activity of SIX2 may well represent a prosurvival pathway for malignant WT cells analogous to the condensing MM. Other molecular factors important in renal progenitor cell survival and function have been implicated in WT pathogenesis, including the signal transducer STAT1 $[18,19]$ and the chemokine receptor CXCR2 [20].

Although our data provide no mechanistic insight into the role of SIX2 in WT, Aiden et al [21] recently characterized the chromatin profile of WT specimens and identified a large active chromatin domain that overlaps the SIX2 locus, suggesting dysregulated epigenetic control of SIX2 in WT. In addition, other developmentally relevant genes including GDNF, SOX11, EYA1, and OSR1 were similarly identified [21]. SIX2 promoter hypomethylation has also been detected in a series of primary WT [22]. Taken together, these results suggest that epigenetic mechanisms may function as critical regulators of SIX2 expression in WT and raise the question of whether WT cells exploit developmental pathways of selfrenewal for tumor initiation and growth. Indeed, previous work from our laboratory supports the role of CITED1 in tumor cell self-renewal as a regulator of cell proliferation and tumorigenesis $[10,11]$. Ongoing efforts in our laboratory are focused on determining the functional significance of SIX2 independent of and in combination with CITED1 in WT. Perhaps disrupting these transcriptional programs could attenuate cancer cell self-renewal and provide an avenue for novel WT therapies.

Although similar expression of SIX2 and CITED1 was detected across the aforementioned WT features, rare instances were observed in which a given tumor region expressed either one or the other protein alone. Heterogeneous expression of critical embryonal transcriptional pro- grams within a given WT is not unprecedented. In fact, activation of canonical Wnt signaling as estimated by nuclear localization of $\beta$-catenin is often detected in as few as $5 \%$ of cells within a given tumor $[23,24]$. Like SIX2 and CITED1 in the current study, nuclear localization of $\beta$-catenin has not been associated with any specific pathogenic feature of WT and may also be important in WT cancer cell survival or tumorigenesis. Although differential expression of SIX2 and CITED1 did not associate with any specific pathogenic feature in the WTs provided for this study, perhaps these expression patterns link a given WT to a specific progenitor cell population or temporal stage in kidney development. For example, a tumor with high SIX2 and low CITED1 expression could originate from the population of $\mathrm{SIX}^{+}$/ CITED $^{-}$cells detected in the ventral cap mesenchyme and in pretubular aggregates, as shown previously $[4,13]$. Alternatively, this tumor could originate from early nephrogenesis when SIX2 expression in the cap mesenchyme has commenced, but CITED1 expression has not [5]. In this way, unique expression patterns may link a given WT to a specific stage of kidney development and thereby expose other candidate targets to promote terminal differentiation of WT.

The current study results differ slightly from our previously published observations that showed CITED1 expression associated with stage IV disease in WT [10]. This phenomenon is likely true in favorable histology WT but was not observed in the current investigation, possibly because of the high proportion of unfavorable disease characteristics examined herein. Furthermore, the tumors examined in the current study were predominantly triphasic, whereas our previous cohort contained a high proportion of blastemal predominant tumors, which may express increased levels of CITED1 given its propensity for strong blastemal detection 
by immunohistochemistry. Additional limitations of this study include the potential for sampling error, as small regions from otherwise large tumors were analyzed. In addition, this study was limited by the variability inherent in obtaining specimens from a tissue bank (Children's Oncology Group), whose source material may be subject to inconsistent quality control with respect to specimen acquisition, tissue fixation, and processing.

Our results support recent evidence that a complex network of developmentally regulated transcriptional programs contributes to Wilms' tumorigenesis. Known genetic mutations occur in less than $50 \%$ of WTs and have been principally applicable as prognostic determinants [25]. In light of the limited applicability of known genetic mutations to the development of specific and novel cell-based therapies, perhaps transcriptional activators that promote cellular self-renewal will provide fertile ground for broadly applicable therapeutic discoveries in WT.

\section{Acknowledgments}

This work was supported by the Section of Surgical Sciences and the Ingram Cancer Center of the Vanderbilt Medical Center, by the National Cancer Institute (NCI) grant 5T32CA106183-06A1 (AJM) and by NCI grant 4R00CA135695-03 (HNL). We would like to acknowledge the excellent services of the Children's Oncology Group Biopathology Center, which were instrumental in specimen acquisition and data analysis and also the NCI-funded Human Tissue Acquisition and Pathology Shared Resource at Vanderbilt (grant P30 CA68485).

\section{References}

[1] Li CM, Guo M, Borczuk A, et al. Gene expression in Wilms tumor mimics the earliest committed stage in the metanephric mesenchymalepithelial transition. Am J Pathol 2002;160:2181-90.

[2] Davidoff AM. Wilms' tumor. Curr Opin Pediatr 2009;21:357-64.

[3] Saxen L, Sariola H. Early organogenesis of the kidney. Pediatr Nephrol 1987;1:385-92.

[4] Hendry C, Rumballe B, Moritz K, et al. Defining and redefining the nephron progenitor population. Pediatr Nephrol 2011.

[5] Kobayashi A, Valerius MT, Mugford JW, et al. Six2 defines and regulates a multipotent self-renewing nephron progenitor population throughout mammalian kidney development. Cell Stem Cell 2008;3: 169-81.

[6] Boyle S, Misfeldt A, Chandler KJ, et al. Fate mapping using Cited1CreERT2 mice demonstrates that the cap mesenchyme contains selfrenewing progenitor cells and gives rise exclusively to nephronic epithelia. Dev Biol 2008:313:234-45.

[7] Boyle S, Shioda T, Perantoni AO, et al. Cited1 and Cited2 are differentially expressed in the developing kidney but are not required for nephrogenesis. Dev Dyn 2007;236:2321-30.

[8] Self M, Lagutin OV, Bowling B, et al. Six2 is required for suppression of nephrogenesis and progenitor renewal in the developing kidney. EMBO J 2006;25:5214-28.
[9] Rivera MN, Haber DA. Wilms' tumour: connecting tumorigenesis and organ development in the kidney. Nat Rev Cancer 2005;5:699-712.

[10] Lovvorn HN, Westrup J, Opperman S, et al. CITED1 expression in Wilms' tumor and embryonic kidney. Neoplasia 2007;9:589-600

[11] Lovvorn III HN, Boyle S, Shi G, et al. Wilms' tumorigenesis is altered by misexpression of the transcriptional co-activator, CITED1. J Pediatr Surg 2007;42:474-81.

[12] Shi G, Boyle SC, Sparrow DB, et al. The transcriptional activity of CITED1 is regulated by phosphorylation in a cell cycle-dependent manner. J Biol Chem 2006;281:27426-35.

[13] Mugford JW, Yu J, Kobayashi A, et al. High-resolution gene expression analysis of the developing mouse kidney defines novel cellular compartments within the nephron progenitor population. Dev Biol 2009;333:312-23.

[14] Huff V. Wilms' tumours: about tumour suppressor genes, an oncogene and a chameleon gene. Nat Rev Cancer 2011;11:111-21.

[15] Dressler GR, Douglass EC. Pax-2 is a DNA-binding protein expressed in embryonic kidney and Wilms tumor. Proc Natl Acad Sci USA 1992;89:1179-83.

[16] Paik S, Rosen N, Jung W, et al. Expression of insulin-like growth factor-II mRNA in fetal kidney and Wilms' tumor. An in situ hybridization study. Lab Invest 1989;61:522-6.

[17] $\mathrm{Hu} \mathrm{Q}$, Gao F, Tian W, et al. Wt1 ablation and Igf2 upregulation in mice result in Wilms tumors with elevated ERK1/2 phosphorylation. J Clin Invest 2011;121:174-83.

[18] Timofeeva OA, Plisov S, Evseev AA, et al. Serine-phosphorylated STAT1 is a prosurvival factor in Wilms' tumor pathogenesis. Oncogene 2006;25:7555-64.

[19] Wang H, Yang Y, Sharma N, et al. STAT1 activation regulates proliferation and differentiation of renal progenitors. Cell Signal 2010;22:1717-26.

[20] Levashova ZB, Sharma N, Timofeeva OA, et al. ELR+-CXC chemokines and their receptors in early metanephric development. J Am Soc Nephrol 2007;18:2359-70.

[21] Aiden AP, Rivera MN, Rheinbay E, et al. Wilms tumor chromatin profiles highlight stem cell properties and a renal developmental network. Cell Stem Cell 2010;6:591-602.

[22] Metsuyanim S, Pode-Shakked N, Schmidt-Ott KM, et al. Accumulation of malignant renal stem cells is associated with epigenetic changes in normal renal progenitor genes. Stem Cells 2008;26:1808-17.

[23] Koesters R, Niggli F, von Knebel Doeberitz M, et al. Nuclear accumulation of beta-catenin protein in Wilms' tumours. J Pathol 2003;199: 68-76.

[24] Ehrlich D, Bruder E, Thome MA, et al. Nuclear accumulation of betacatenin protein indicates activation of wnt signaling in chemically induced rat nephroblastomas. Pediatr Dev Pathol 2010;13:1-8.

[25] Ruteshouser EC, Robinson SM, Huff V. Wilms tumor genetics: mutations in WT1, WTX, and CTNNB1 account for only about onethird of tumors. Genes Chromosomes Cancer 2008;47:461-70.

\section{Discussion}

Discussant, Dr Paul Losty (Liverpool, United Kingdom): This is very interesting work. Have you looked for colocalizations of stem cell markers?

Response, Dr Murphy: At this point, we can draw an analogy between the expression of these 2 genes in Wilms' tumor and the fact that the expression of these 2 genes in development determines the stem cell phenotype. We've characterized in separate studies the functional role of CITED1 in Wilms' tumor, which seems to promote tumor 
cell self-renewal and tumorigenesis in vitro and in vivo. We're currently working out the conditions to explore those roles with SIX2. For now, we can draw an analogy to development. We have not proven that those are stem cells in the tumor themselves.

Discussant, Dr Mary Brandt (Houston, TX): You showed us the tumor and you showed us the fetal kidney. How much is this expressed in the adult or developed kidney?

Response, Dr Murphy: That's an excellent question. These 2 genes are off by the time nephrogenesis concludes, so they are not expressed in the developed kidney whatsoever. They turn off at the very terminal stages of nephrogenesis. There's actually a little bit of temporal distinction between the 2 as well, SIX2 comes on earlier in development and is present until the conclusion of nephrogenesis, whereas CITED1 is expressed in a more linear manner, so these are often noted in the adult kidney.

Discussant, Dr Brandt: If you took a kidney with Wilms' tumor and stained it and did all these studies in the normal kidney as well, there would be none in the normal and how much in the tumor?

Response, Dr Murphy: That's correct, and the interesting thing would be to look at the intermediate between the 2 which would be nephrogenic rests and in the limited number of specimens that we have with nephrogenic rests, they do stain positive for CITED1 and SIX2 suggesting that persistence of these stem cells in the precursor lesion to Wilms' tumor also expresses these 2 key genes. 